\title{
Preoperative levels of inflammatory biomarkers in patients with bilateral inferior turbinate hypertrophy.
}

\author{
Akif Gunes $^{1 *}$, Mehmet Fatih Karakus², Nurcan Akbas Gunes ${ }^{3}$, Murad Mutlu ${ }^{4}$ \\ ${ }^{1}$ Department of Otorhinolaryngology, Eskisehir State Hospital, Eskisehir, Turkey \\ ${ }^{2}$ Department of Otorhinolaryngology, Ankara Numune Education and Research, Ankara, Turkey \\ ${ }^{3}$ Yeni Family Health Center, Eskisehir, Turkey \\ ${ }^{4}$ Department of Otorhinolaryngology, Diskapi Yildirim Beyazit Education and Research Hospital, Ankara, Turkey
}

\begin{abstract}
Objective: The aim of this study was to evaluate preoperative inflammatory biomarkers in patients who underwent surgical treatment with the indication of bilateral inferior turbinate hypertrophy.

Materials and methods: Eighty patients who applied to our otorhinolaryngology clinic with complaints of chronic nasal obstruction and 79 clinically healthy individuals were included in the study. This was a retrospective study. Evaluation was performed on the patient records. Hemogram values of 80 patients were compared with 79 clinically healthy individuals. Both groups were compared with respect to WBC (White Blood Cell), L (Lymphocyte), N (Neutrophil), PLT (Platelet), M (Monocyte) counts, N/L (Neutrophil/Lymphocyte ratio), PLT/L (Platelet/Lymphocyte ratio), M/L (Monocyte/Lymphocyte ratio), M/WBC (Monocyte/leucocyte ratio), and N/WBC (Neutrophil/leucocyte ratio). $\mathbf{P}<0.05$ was accepted as the level of statistical significance.

Results: In intergroup evaluation, a statistically significant difference was not found between groups as for WBC, N, and PLT counts. However two groups were statistically significantly different regarding $L$, and $M$ values ( $p=0.026, p=0.000$, and $p<0.05$, respectively). Besides a statistically significant difference was not found between two groups as for N/L, PLT/L, and N/WBC ratios. However a statistically significant difference was observed between both groups regarding $M / L$, and $M / W B C$ ratios $(p=0.000$, $\mathbf{p}=\mathbf{0 . 0 0 0}$, and $\mathbf{p}<0.05$, respectively).

Conclusion: Easily calculated $\mathrm{M} / \mathrm{L}$, and $\mathrm{M} / \mathrm{WBC}$ ratios with low cost can be used as auxiliary parameters in the evaluation of patients with inferior turbinate hypertrophy.
\end{abstract}

Keywords: Turbinate, Neutrophil, Monocyte, Lymphocyte.

\section{Introduction}

Inferior nasal turbinates are anatomic structures with important functions in humidification of air, and adjustment of temperature. Sympathetic and parasympathetic system change dimensions of the turbinate by decreasing, and increasing blood flow in erectile tissue in turbinates according to physiologic requirements [1].

In pathologic conditions as allergic rhinitis or viral infection, the fluid extravasates from capillaries into extracellular space leading to swelling of turbinates $[1,2]$. Generally perennial allergic rhinitis and vasomotor (non-allergic) rhinitis are etiologic factors of chronic nasal obstruction due to inferior turbinate hypertrophy. Turbinate hypertrophy is generally bilateral, and it is secondary to mucosal thickening without hypertrophy of the underlying tissues [1].
In inferior turbinate hypertrophies developed on the background of chronic rhinitis, histopathologically submucosal edema, and cellular infiltration can be seen [1]. In a study histopathological analyses were performed in patients with inferior turbinate hypertrophy, and as a result compared with the control group, a significant increase in inflammatory cell counts was observed in the subepithelial region [3].

In the hemogram analysis of peripheral blood samples, Neutrophil/Lymphocyte ratio (N/L), Platelet/Lymphocyte ratio (PLT/L), Monocyte/Lymphocyte ratio (M/L), Monocyte/ leucocyte ratio $(\mathrm{M} / \mathrm{WBC})$, and Neutrophil/leucocyte ratio $(\mathrm{N} /$ WBC) can be calculated. These ratios were found to be higher in various peripheral diseases, and its possible use in the prognosis, and follow-up of the patients has been reported $[4,5]$. 
In various studies performed $\mathrm{N} / \mathrm{L}$ ratio was evaluated in patients with nasal polyposis, and allergic rhinitis, and significantly higher results were detected. In these patient groups N/L ratio has been reported to be a marker with a lower cost [6].

In literature reviews, a study which evaluated the relationship between N/L, PLT/L, M/L, M/WBC, N/WBC, and bilateral inferior turbinate hypertrophy has not been encountered. In our study, we aimed to evaluate preoperative Neutrophil (N), Lymphocyte (L), Monocyte (M), Platelet (PLT) and leucocyte or White Blood Cell (WBC) counts, and N/L, PLT/L, M/L, M/ $\mathrm{WBC}$, and $\mathrm{N} / \mathrm{WBC}$ ratios in patients who underwent surgical treatment with the indication of bilateral inferior turbinate hypertrophy.

\section{Materials and Methods}

Eighty patients who applied to our otorhinolaryngology clinic with complaints of chronic nasal obstruction between 2012 and 2017 were included in the study. This was a retrospective study. Evaluation was performed on the patient records. The consent of local ethics committee was obtained. The study was conducted in accordance with the guidelines of the Declaration of Helsinki.

The patients who had complaints of nasal discharge, and obstruction, postnasal discharge lasting for more than three months were included in the study. In detailed ear, nose and throat examinations, both during anterior rhinoscopy and nasal endoscopic examination, the patients were evaluated as for the presence of septal deviation, nasal polyps, inflammatory mucosal changes, and condition of turbinates, and surgerynaive patients without any pathology other than bilateral inferior turbinate hypertrophy or those without any improvement in their complaints despite three months of medical treatment were included in the study Besides patients with unilateral turbinate hypertrophy, those with anatomical variations or additional pathology as detected on paranasal sinus Computed Tomography (CT) or cases with acute, chronic sinusitis or septal deviation were excluded from the study.

Hemogram values of 80 patients included in the present study were compared with those of 79 clinically healthy individuals were selected as the control group, who admitted to the hospital for check-up, without any health problems and comorbidities. The control group had an endoscopic exam to check for the absence of sinonasal pathology.

To exclude other systemic diseases, all participants included in the study were excluded from the study who have diabetes mellitus, hypertension, acute coronary artery disease, vasculitis, connective tissue disorder, chronic renal and liver failure.

Plasma glucose, complete blood count, lipid profile, vit B12, electrolyte levels, thyroid hormone levels, blood pressure, hepatitis markers and body temperature of the participants included to this study were analysed and patients with identifiable pathology were excluded.

In both groups, for standardization, hemograms were evaluated by using the results obtained with the same device (Siemens Advia 2120i, Ireland). Both groups were compared with respect to $\mathrm{WBC}, \mathrm{L}, \mathrm{N}, \mathrm{PLT}, \mathrm{M}$ counts, N/L, PLT/L, M/L, $\mathrm{M} / \mathrm{WBC}$ and N/WBC.

\section{Statistical analysis}

Statistical analysis was performed using the Statistical Package for the Social Sciences 18.0 (SPSS, Inc.; Chicago, IL, USA) version for Windows. Descriptive statistics were expressed as mean \pm standard deviation, as the median for continuous variables and as the number of observations (\%) for nominal variables. Bivariate associations were determined using the chi-square test for categorical variables, the Mann-Whitney $U$ test was used for the comparison of non-normally distributed continuous variables. Statistical results with a value of $\mathrm{p}<0.05$ were considered statistically significant.

\section{Results}

A total of 80 patients with a mean age of $28.40 \pm 9.346$ (range, 15- 56) years were included in the study. The study group consisted of 42 male (52.5\%), and 38 female (47.5\%) patients. In the control group 79 healthy individuals were evaluated. Mean age of the control group was $29.58 \pm 6.802$ (range, 18-50 y). Control group consisted of 38 male $(48.1 \%)$, and 41 female $(51.9 \%)$ patients (Table 1$)$.

In intergroup evaluation, a statistically significant difference was not found between groups as for WBC, N, and PLT counts. However two groups were statistically significantly different regarding $\mathrm{L}$, and $\mathrm{M}$ values $(\mathrm{p}<0.05)$ (Table 2$)$.

Besides a statistically significant difference was not found between two groups as for N/L, PLT/L, and N/WBC ratios. However a statistically significant difference was observed between both groups regarding $\mathrm{M} / \mathrm{L}$, and $\mathrm{M} / \mathrm{WBC}$ ratios $(\mathrm{p}<0.05)$ (Table 3).

Table 1. Age and gender data of both groups.

\begin{tabular}{llllll}
\hline & \multicolumn{1}{l}{ Age } & & Gender & \\
\cline { 2 - 5 } & Minimum & Maximum & Mean \pm SD & Male (\%) & Female (\%) \\
\hline Patient group & 15 & 56 & $28.40 \pm 9.346$ & $42(52.5 \%)$ & $38(47.5 \%)$ \\
\hline
\end{tabular}


Control group 18 50 $29.58 \pm 6.802$ $38(48.1 \%)$ $41(51.9 \%)$

Table 2. Mean WBC, N, L, PLT, $M$ value and p-values (WBC: White Blood Cell; N: Neutrophil; L: Lymphocyte; PLT: Platelet; M: Monocyte).

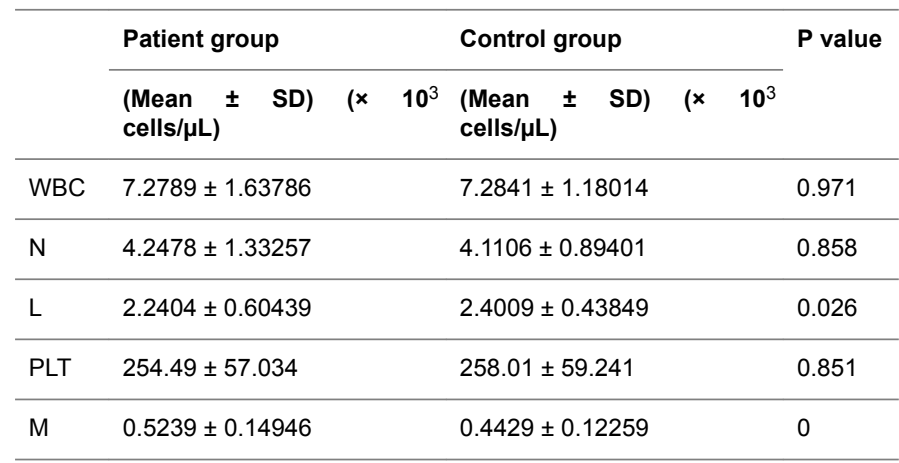

Table 3. Mean $N / L, P L T / L, M / L, N / W B C$, and $M / W B C$ levels and $p$ values (N/L: Neutrophil/Lymphocyte ratio; PLT/L: Platelet) Lymphocyte ratio; M/L: Monocyte/Lymphocyte ratio; N/WBC: Neutrophil/White Blood Cell ratio; M/WBC: Monocyte/white blood cell ratio).

\begin{tabular}{llll}
\hline & Patient group & Control group & \multirow{2}{*}{ P value } \\
\cline { 2 - 3 } & (Mean \pm SD) & (Mean \pm SD) & \\
\hline $\mathrm{N} / \mathrm{L}$ & $2.0200 \pm 0.84906$ & $1.7529 \pm 0.44295$ & 0.103 \\
\hline $\mathrm{PLT} / \mathrm{L}$ & $122.39 \pm 46.36569$ & $110.72 \pm 33.02870$ & 0.157 \\
\hline $\mathrm{M} / \mathrm{L}$ & $0.2459 \pm 0.08969$ & $0.1876 \pm 0.05564$ & 0 \\
\hline $\mathrm{N} / \mathrm{WBC}$ & $0.5771 \pm 0.08427$ & $0.5613 \pm 0.05666$ & 0.158 \\
\hline $\mathrm{M} / \mathrm{WBC}$ & $0.0729 \pm 0.01826$ & $0.0609 \pm 0.01320$ & 0 \\
\hline
\end{tabular}

\section{Discussion}

Inferior turbinate hypertrophy is one of the most important causes of nasal obstruction. Bilateral inferior turbinate hypertrophies develop secondary to allergic or non-allergic rhinitis, while unilateral inferior turbinate hypertrophies develop secondary to congenital etiologies or septum deviations [7]. When histopathological sections of the specimens obtained from inferior turbinate hypertrophies which occurred on the background of chronic rhinitis are examined, submucosal edema, and cellular infiltration, thickening of the subepithelial basal membrane, and hyalinization, squamous metaplasia which can occur concomitantly with fibroplastic proliferation of stroma, ciliar desquamations, dilation of blood vessels, and hypersecretion of mucous glands can be seen. Following development of fibrosis, response to medical therapy weakens [3]. We performed surgical treatment on 80 patients with bilateral inferior turbinate hypertrophy who did not respond to 3 months of medical therapy.

In a study performed, when compared with the control group, in the histopathological examination of the specimens obtained from the patients with inferior turbinate hypertrophy, increase in inflammatory cells consisting of lymphocytes, monocytes, plasma cells, neutrophils, eosinophils, and mast cells were demonstrated [3]. In various studies, levels of inflammatory cells were calculated, and the ratios of these inflammatory cells with each other were compared with those of the control group. The authors concluded that the estimated values could be simple and inexpensive indicators of inflammation [6].

In our study in patients with turbinate hypertrophy in whom inflammatory process was demonstrated histopathologically, we evaluated preoperative $\mathrm{WBC}, \mathrm{L}, \mathrm{N}, \mathrm{PLT}, \mathrm{M}$ values, and $\mathrm{N} / \mathrm{L}, \mathrm{PLT} / \mathrm{L}, \mathrm{M} / \mathrm{L}, \mathrm{M} / \mathrm{WBC}$, and $\mathrm{N} / \mathrm{WBC}$ ratios [3]. We reviewed the literature, and didn't encounter any similar study conducted in patients with turbinate hypertrophy.

In a study performed on the patients diagnosed as nasal polyps, leucocyte, and platelet counts were estimated, however significant changes were not seen when compared with those of the control group [8]. However, in another study statistically significantly higher leucocyte, and platelet counts were detected in patients with nasal polyps [6]. In our study we could not find significant results as for leukocyte, and platelet values.

$\mathrm{N} / \mathrm{L}$ ratio increases in systemic inflammation, in some diseases $[4,5]$. In a different study, the authors demonstrated significance of N/L ratio in the follow-up, and prognosis of the patients with different malignancies [9].

In a study performed on patients with sudden hearing loss $\mathrm{N} / \mathrm{L}$ ratio was found to be statistically significantly higher when compared with the control group, and the authors concluded that increase in $\mathrm{N} / \mathrm{L}$ ratio can be evaluated as a poor prognostic factor [10]. In another study performed on patients with facial paralysis, neutrophil counts, and N/L ratio were compared with those of the control group, and both neutrophil counts, and N/L ratio were statistically significantly higher in the patient group [11]. However, we couldn't find a significant difference between both groups as for neutrophil counts, and N/L ratios.

Platelet count is one of the markers which demonstrate thrombocytic activation, and function. In inflammation, an increase in platelet counts can be seen [12]. Similar to N/L ratio, $\mathrm{PLT} / \mathrm{L}$ ratio is also a marker of chronic inflammation. In a study performed on 619 patients with myocardial infarction, it was shown that higher PLT/L value increased mortality and dual antiplatelet treatment of patients with higher PLT/L ratio decreased mortality when compared with single antiplatelet treatment [13]. In our study we couldn't find a statistically significant difference between groups with respect to PLT/L ratios.

Monocytes are accepted as inflammatory biomarkers [14]. In a study, in patients with coronary artery disease $\mathrm{M} / \mathrm{L}$ ratio was evaluated and it was concluded that increased $\mathrm{M} / \mathrm{L}$ ratio was associated with increase in depressive symptoms. In the same 
study, increase in $\mathrm{M} / \mathrm{L}$ ratio was demonstrated to be directly proportional to increase in neuroendocrine-sympathetic activity, onset, and progression of atherosclerosis, and the authors concluded that $\mathrm{M} / \mathrm{L}$ ratio can be used as an inflammatory marker in the follow-up of the patients [15]. In our study we couldn't find a statistically significant difference between both groups as for $\mathrm{N} / \mathrm{WBC}$ ratio. However we obtained significant results when we compared monocyte counts, $\mathrm{M} / \mathrm{L}$, and $\mathrm{M} / \mathrm{WBC}$ ratios with those of the control group.

Our study limitation was that we only analysed preoperative hemogram values in patients with inferior turbinate hypertrophy on whom we performed surgery. Patients with bilateral inferior turbinate hypertrophy can be evaluated during postoperative period, and $\mathrm{WBC}, \mathrm{L}, \mathrm{N}, \mathrm{PLT}, \mathrm{M}$ values can be compared with N/L, PLT/L, M/L, M/WBC, and N/WBC ratios or patient can be grouped based on the surgery performed, and short-, and long-term effects of surgical technique on inflammation can be evaluated. Besides pre- and postoperative acoustic rhinometric assessments can be used to compare levels of inflammation. Pre- and postoperative Visual Analog Scale (VAS) measurements of the patients can be compared with markers of inflammation. Our study can be guidance for further studies.

In conclusion, when we compared the patients whom we operated because of bilateral inferior turbinate hypertrophy with the control group, regarding preoperative $\mathrm{M}$, and $\mathrm{L}$ counts, $\mathrm{M} / \mathrm{L}$ ratio, and $\mathrm{M} / \mathrm{WBC}$ ratio we obtained significant results. We think that easily calculated $\mathrm{M} / \mathrm{L}$, and $\mathrm{M} / \mathrm{WBC}$ ratios with low cost can be used as auxiliary parameters in the evaluation of patients with inferior turbinate hypertrophy.

\section{Acknowledgement}

The authors declare no competing interest. No financial support was received for this paper.

\section{References}

1. Ophir DE, Shapira AA, Marshak GS. Total inferior turbinectomy for nasal airway obstruction. Arch Otolaryngol 1995; 11: 93-95.

2. Robbins SL. Pathologic basis of disease (3rd edn.). Phil 1989: 763-764.

3. Berger G, Gass S, Ophir D. The histopathology of the hypertrophic inferior turbinate. Arch Otolaryngol Head Neck Surg 2006; 132: 588-594.

4. Bhat T, Teli S, Rijal J, Bhat H, Raza M, Khoueiry G. Neutrophil to lymphocyte ratio and cardiovascular diseases: a review. Expert Rev Cardiovasc Ther 2013; 11 : 55-59.

5. Proctor MJ, McMillan DC, Morrison DS, Fletcher CD, Horgan PG, Clarke SJ. A derived neutrophil to lymphocyte ratio predicts survival in patients with cancer. Br J Cancer 2012; 107: 695-699.

6. Dogan A, Kursat MO, Sabri K, Aykut I, Mehmi C, Serdar E. New predictive parameters of nasal polyposis: neutrophil to lymphocyte ratio and platelet to lymphocyte ratio. Kulak Burun Bogaz Ihtis Derg 2015; 25: 97-101.

7. Egeli E, Demerci L, Yazici B, Harputlouglu U. Evaluation of the inferior turbinate in patients with deviated nasal septum by using computed tomography. Laryngoscope 2004; 114: 113-117.

8. Aktas G, Sit M, Tekce H, Alcelik A, Savli H, Simsek T. Mean platelet volume in nasal polyps. West Indian Med J 2013; 62: 515-518.

9. Xin S, Gui-Ming Z, Xiao-Cheng M, Lei L, Bin L, DongYue C. Comparison of preoperative neutrophillymphocyte, lymphocyte-monocyte, and plateletlymphocyte ratios in patients with upper urinary tract urothelial carcinoma undergoing radical nephroureterectomy. Onco Targets Ther 2016; 9: 1399-1407.

10. Ulu S, Ulu MS, Bucak A, Ahsen A, Yucedag F, Aycicek A. Neutrophil-to-lymphocyte ratio as a new, quick, and reliable indicator for predicting diagnosis and prognosis of idiopathic sudden sensorineural hearing loss. Otol Neurotol 2013; 34: 1400-1444.

11. Bucak A, Ulu S, Oruc S, Yucedag F, Tekin MS, Karakaya F. Neutrophil-to-lymphocyte ratio as a novel potential marker for predicting prognosis of Bell palsy. Laryngoscope 2014; 124: 1678-1681.

12. Unnikrishnan D, Jun J, Polotsky V. Inflammation in sleep apnea: an update. Rev Endocr Metab Disord 2015; 16: 25-34.

13. Azab B, Shah N, Akerman M, McGinn JT. Value of platelet/lymphocyte ratio as a predictor of allcause mortality after non-ST-elevation myocardial infarction. J Thromb Thrombolysis 2012; 34: 326-334.

14. Jaipersad AS, Lip GY, Silverman S, Shantsila E. The role of monocytes in angiogenesis and atherosclerosis. J Am Coll Cardiol 2014; 63: 1-11.

15. Serfozo G, Horvath T, Foldesi I, Rafael B, von Kanel R, Keresztes $M$. The monocyte-to lymphocyte ratio correlates with psycho-neuro-inflammatory factors in patients with stable coronary artery disease. Neuroimmunomodulation 2016.

\section{${ }^{*}$ Correspondence to}

Akif Gunes

Department of Otorhinolaryngology

Eskisehir State Hospital

Turkey 